\title{
Acknowledgement to Reviewers of Pharmaceuticals in 2015
}

\section{Pharmaceuticals Editorial Office}

Published: 21 January 2016

MDPI AG, Klybeckstrasse 64, CH-4057 Basel, Switzerland; pharmaceuticals@mdpi.com

The editors of Pharmaceuticals would like to express their sincere gratitude to the following reviewers for assessing manuscripts in 2015.

We greatly appreciate the contribution of expert reviewers, which is crucial to the journal's editorial decision-making process. Several steps have been taken in 2015 to thank and acknowledge reviewers. Good, timely reviews are rewarded with a discount off their next MDPI publication. By creating an account on the submission system, reviewers can access details of their past reviews, see the comments of other reviewers, and download a letter of acknowledgement for their records. In addition, MDPI has launched a collaboration with Publons, a website that seeks to publicly acknowledge reviewers on a per journal basis. This is all done, of course, within the constraints of reviewer confidentiality. Feedback from reviewers shows that most see their task as a voluntary and mostly unseen work in service to the scientific community. We are grateful to our reviewers for the contribution they make.

$\begin{array}{lll}\text { Arendash, Gary W. } & \text { Hulli, Roger } & \text { Reau, Nancy } \\ \text { Armon, Robert } & \text { Hwang, William Ying Khee } & \text { Reddy, Nishitha } \\ \text { Bao, Yongpin } & \text { Jain, Prashant } & \text { Rhee, Frits Van } \\ \text { Beaulieu, Martin } & \text { Jenssen, Håvard } & \text { Rhodes, Jonathan } \\ \text { Berger, Elizabeth } & \text { Katsila, Theodora } & \text { Robertson, J. David } \\ \text { Bishop, Barney } & \text { Khiari, Zied } & \text { Romeo, Giovanna } \\ \text { Blyth, Emily } & \text { Kinoshita, Takayoshi } & \text { Roque, Dana M. } \\ \text { Brackman, Gilles } & \text { Kolodkin-Gal, Ilana } & \text { Ryan, Robert P. } \\ \text { Brynildsen, Mark P. } & \text { Koudelka, Gerald } & \text { Sadekar, Shraddha } \\ \text { Cabot, Myles } & \text { Lai, Shih-Wei } & \text { Sadelain, Michel } \\ \text { Cai, Jianfeng } & \text { Larraza, Sebastian } & \text { Said, Ahmed M. } \\ \text { Canna, Scott W. } & \text { Lee, Hsin-Chen } & \text { Schamel, Wolfgang W.A. } \\ \text { Carta, Fabrizio } & \text { Lévesque, Céline M. } & \text { Schibli, Roger } \\ \text { Čeřovský, Václav } & \text { Liao, Daiqing } & \text { Schoenhagen, Paul } \\ \text { Chrubasik, Sigrun } & \text { Ling, Jun } & \text { Schwan, William R. } \\ \text { Cranfield, Charles } & \text { Lorenz, Kristina } & \text { Scotet, Emmanuel } \\ \text { Cron, Randy Q. } & \text { Mancardi, Daniele } & \text { Sima, Jian } \\ \text { Dallinger, Doris } & \text { Marques, Claudia } & \text { Simmonds, Monique S.J. } \\ \text { Dehaye, Jean-Paul } & \text { Masclee, G. M. C. } & \text { Sulzmaier, Florian J. } \\ \text { Delisle, Jean-Sébastien } & \text { McBain, Andrew } & \text { Takeda, Shin'ichi } \\ \text { Di Bonaventura, Giovanni } & \text { Mehta, Amitkumar } & \text { Tang, Gong } \\ \text { Domínguez Álvarez, Enrique } & \text { Mehta, Akul Y. } & \text { Tang, Yu } \\ & & \\ & & \end{array}$


Dow, Max

Duman, Ronald

Eppard, Elisabeth

Fagerlund, Malin Jonsson

Fernández, Lucía

Frost, Christopher G.

Frost, Sofia H. L.

Fukunaga, Kaori

Garcia, Pilar

Gellerman, Gary

Ghosh, Biswarup

Gourley, Shannon L.

Gøtze, Jens Peter

Gray, Elaine

Gursoy, Ulvi K.

Hazan, Ronen

Hofmann, Johann

Horne, Seth

Huang, Li

Huang, Tien L.

Hudecek, Michael
Mineishi, Shin

Minor, Thomas R.

Mitra, Abhisek

Mollereau, Bertrand

Moser, Bernhard

Moskowitz, Alison J.

Mulloy, Barbara

Nagant, Carole

Nelson, Daniel

Neri, Marcella

Nicholl, Iain

Oratz, Ruth

Oxenkrug, Gregory F.

Pelicano, Hélène

Pereira, Leonel

Piazza, Gary

Pouget, Jean-Pierre

Poznański, Jarosław

Prykhodko, Olena

Rajendran, Praveen

Rasche, Leo
Thevissen, Karin

Titz, Alexander

Tomkinson, Nicholas

Tsantrizos, Youla S.

Uehara, Tomoya

van Hoek, Monique L.

van Kaer, Luc

Vázquez, Julio

Villalobos, Carlos

Wei, Dongshan

Witting, Paul

$\mathrm{Wu}$, Chung-Hsin

Xiang, Yang $\mathrm{K}$.

Xiaoming, Xie

Xie, Bin

Yeoman, Caarl

Young, Jette F.

Zalutsky, Michael R.

Zhang, P.

Ziegelbauer, Joseph M.

(C) 2016 by the authors; licensee MDPI, Basel, Switzerland. This article is an open access article distributed under the terms and conditions of the Creative Commons by Attribution (CC-BY) license (http://creativecommons.org/licenses/by/4.0/). 